\title{
Universal Usability: Pushing Human-Computer Interaction Research to Empower Every Citizen
}

\author{
Ben Shneiderman \\ Department of Computer Science, \\ Human-Computer Interaction Laboratory, \\ Institute for Advanced Computer Studies \& \\ Institute for Systems Research \\ University of Maryland, College Park, MD 20742 USA
}

July 31, 1999

"I feel... an ardent desire to see knowledge so disseminated through the mass of mankind that it may...reach even the extremes of society: beggars and kings."

-- Thomas Jefferson, Reply to American Philosophical Society, 1808

In a fair society, all individuals would have equal opportunity to participate in, or benefit from, the use of computer resources regardless of race, sex, religion, age, disability, national origin or other such similar factors.

-- ACM Code of Ethics

Position Paper for National Science Foundation \& European Commission meeting on humancomputer interaction research agenda, June 1-4, 1999, Toulouse, France. To be published in book form.

\section{Introduction}

The goal of universal access to information and communications services is compelling. It has united hardworking Internet technology promoters, telecommunications business leaders, and government policy makers. Their positive motivations include innovative visions, corporate opportunities, and social goods respectively, although critics see unreasonable zeal for technology, pursuit of high profit margins, and regulatory excesses or omissions.

Each sector is working hard to contribute what they see as valuable, while trying to respond to critics. Most technology infrastructure developers seek to accommodate high volumes of use, reliably and rapidly even at peak periods, while ensuring security. Most service providers strive to develop popular communications, e-commerce, healthcare, education, and other services, while ensuring profitability. Most government officials struggle to provide safety for consumers, freedom of speech, and privacy protection, while supporting voluntary regulation plans.

HCIL Technical Report No. 99-17 (July 1999); http://www.cs.umd.edu/hcil 
Even if all of these professions succeed in their endeavors and the economies of scale bring low costs, computing researchers will still have much work to do. They will have to deal with the difficult question: How can information and communications services be made usable for every citizen? Designing for experienced frequent users is difficult enough, but designing for a broad audience of unskilled users is a far greater challenge. Scaling up from a listserv for 100 software engineers to 100,000 schoolteachers to $100,000,000$ registered voters will take inspiration and perspiration.

Designers of older technologies such as postal services, telephones, and television have reached the goal of universal usability, but computing technology is still too hard to use for many people (Shneiderman, 1998). One survey of 6000 computer users found an average of 5.1 hours per week wasted in trying to use computers. More time is wasted in front of computers than on highways. The frustration and anxiety of users is growing, and the number of non-users is still high. Low-cost hardware, software, and networking will bring in many new users, but interface and information design improvements are necessary to achieve higher levels of access.

We can define universal usability as having more than $90 \%$ of all households as successful users of information and communications services at least once a week. A 1998 survey of US households shows that $42 \%$ have computers and $26 \%$ use Internet-based email or other services (NTIA, 1999). The percentage declines in poorer and less educated areas within the US and in many countries around the world. Cost is an issue for many, but hardware limitations, the perceived difficulty, and lack of utility discourages others. If we are to meet the goal of universal usability, then we will have to directly address usability design issues.

This paper presents a research agenda based on three challenges in attaining universal usability for web-based and other services:

- Technology variety: Supporting a broad range of hardware, software, and network access

- User diversity: Accommodating users with different skills, knowledge, age, gender, handicaps, literacy, culture, income, etc.

- Gaps in user knowledge: Bridging the gap between what users know and what they need to know

This list may not be complete but it addresses important issues that need attention. Research devoted to these challenges will have a broad range of benefits for first time, intermittent and frequent users.

The term universal access is usually linked to the US Communications Act of 1934 covering telephone, telegraph, and radio services. It sought to ensure "adequate facilities at reasonable charges," especially in rural areas and prevent "discrimination on the basis of race, color, religion, national origin, or sex." The term universal access has been applied to computing services, but the greater complexity of computing services means that access is not sufficient to ensure successful usage. Therefore universal usability has emerged as an important issue and a topic for computing research. The complexity emerges, in part, from high degree of 
interactivity that is necessary for information exploration, commercial applications, and creative activities. The Internet is compelling because of its support for interpersonal communications and decentralized initiatives: entrepreneurs can open businesses, journalists can start publications, and citizens can organize political movements.

The increased pressure for universal access and usability is a happy byproduct of the growth of the Internet. Since services such as e-commerce, communication, education, healthcare, finance, and travel are expanding and users are becoming dependent on them, there is a strong push to ensure that the widest possible audience can participate. Another strong argument for universal usability comes from those who provide access to government information (such as the US Library of Congress' THOMAS system to provide full texts of bills before the Congress) and the movement towards citizen services at federal, state, and local levels. These services include tax information and filing, social security benefits, passports, licensing, recreation and parks, and police and fire departments. Another circle of support includes employment agencies, training centers, mental health clinics, parent-teacher associations, public interest groups, community services, and charitable organizations. The enormous potential social good from universal usability creates a grand opportunity for the computing profession.

Critics of information technology abound, but often they focus on the creation of an informationpoor minority, or worse, Internet apartheid. Although the gap in Internet usage has been declining between men and women, and between old and young, the gap is growing between rich and poor and between well and poorly educated. Less well documented is the continuing separation between cultural and racial groups, and the low rates of usage by disadvantaged users whose unemployment, homelessness, poor health, or cognitive limitations raise further barriers (Silver, 1999). Ambitious pursuit of universal access and usability will counter some of their legitimate concerns and help create more effective technologies with more effective support systems for users.

There are other criticisms of information and communications systems that should also be heard by technology promoters. These include concerns about breakdown of community social systems, alienation of individuals that leads to crime and violence, loss of privacy, expansion of bureaucracies, and inadequate attention to potential failures (such as the year 2000 problems or loss of power/data). Open public discussion of these issues by way of participatory design strategies and Social Impact Statements (Shneiderman and Rose, 1997) might reduce negative and unanticipated side effects.

Technology enthusiasts can be proud of what has been accomplished and how many successful Internet users there are, but deeper insights will come from understanding the problems of frustrated unsuccessful users, and of those who have turned away or stayed away. Each step to broaden participation and reach these forgotten users by providing useful and usable services will bring credit to our profession. A necessary first step is to formulate a research agenda.

\section{Previous Research Agendas}


There is growing attention to the computing research issues related to universal access and usability. The thoughtful and focused Rand Corporation report on Universal Access to Email (Anderson, Bikson, Law and Mitchell, 1995) made it clear that "better understanding of the capabilities and limitations of current user-computer interfaces is needed." Similarly, when the National Academy of Science/National Research Council convened a panel on every-citizen interfaces, they recommended "an aggressive research program, funded by government and private sources, that examines both the human performance side of interfaces and the interface technologies, current and potential (CSTB, 1997)."

During a well-financed, but controversial study of 48 Pittsburgh area homes, 133 participants received computers, free network connections, training, and assistance with problems. Even in such optimal conditions a central limitation was the difficulties that users experienced with the services (Kraut, Scherlis, Mukhopadhyay, Manning, and Kiesler, 1996). The researchers wrote "even the easiest-to-use computers and applications pose significant barriers to the use of online services...even with help and our simplified procedure, HomeNet participants had trouble connecting to the Internet."

As attention to the issue of universal access and usability has grown, frameworks for analyzing problems have appeared. Clement and Shade (1999) suggest seven layers of analysis: carriage facilities, devices, software tools, content services, service/access provision, literacy/social facilitation, and governance. They see usability as a problem, especially for users with handicaps, and encourage consideration of the wide range of users and needs. Universal usability is sometimes tied to meeting the needs of users who are disabled or work in disabling conditions. This is an important direction for research that is likely to benefit all users. The adaptability needed for users with diverse physical, visual, auditory, or cognitive handicaps is likely to benefit users with differing preferences, tasks, hardware, etc. (Glinert and York, 1992; Newell, 1995; Laux, McNally, Paciello, and Vanderheiden, 1996). Plasticity of the interface and presentation independence of the contents both contribute to universal usability.

The forthcoming ACM SIGCHI (Special Interest Group on Computer Human Interaction, http://www.acm.org/sigchi) Research Agenda will focus on design of useful, usable and universal user interfaces (Scholtz et al., 1999). SIGCHI has also promoted diversity with its outreach efforts to seniors, kids, teachers, and international groups. The ACM's SIGCAPH (Special Interest Group on Computers and the Physically Handicapped, http://www.acm.org/sigcaph) has long promoted accessibility for disabled users and its ASSETS series of conference proceedings (http://www.acm.org/sigcaph/assets) provide useful guidance. The European conferences on User Interfaces for All (http://www.ics.forth.gr/proj/at-hci/UI4ALL/index.html) also deal with interface design strategies. The Web Accessibility Initiative (http://www.w3.org/WAI) of the World-Wide Web Consortium has a guidelines document with 14 thoughtful content design items to support disabled users, North Carolina State University's Center for Universal Design lists seven key principles (http://www.design.ncsu.edu/cud/), and the University of Wisconsin's TRACE Center offers links to many resources (http://trace.wisc.edu/world). 


\section{A Universal Usability Research Agenda}

This research agenda focuses on three universal usability challenges to designers: technology variety, user diversity, and gaps in user knowledge. Skeptics caution that forcing designers to accommodate low-end technology, low-ability citizens, and low-skilled users will result in a lowest common denominator system that will be less useful to most users. This dark scenario, called dumbing down, is a reasonable fear, but the experience of this author supports a brighter outcome.

I believe that accommodating a broader spectrum of usage situations forces designers to consider a wider range of designs and often leads to innovations that benefit all users. For example, web browsers, unlike word processors, reformat text to match the width of the window. This accommodates users with small displays (narrower than 640 pixels), and provides a nice benefit for users with larger displays (wider than 1024 pixels), who can view more of a web page with less scrolling. Accommodating narrower (less than 400 pixels) or wider (more than 1200 pixels) displays presents just the kind of challenge that may push designers to develop new ideas. For example, they could consider reducing font and image sizes for small displays, moving to a multi-column format for large displays, exploring paging strategies (instead of scrolling), and developing overviews.

A second skeptics' caution, called the innovation restriction scenario, is that attempts to accommodate the low end (technology, ability, or skill) will constrain innovations for the high end. This is again a reasonable caution, but if designers are aware of this concern the dangers seem avoidable. A basic HTML web page accommodates low-end users, and sophisticated user interfaces using Java applets or Shockwave plug-ins can be added for users with advanced hardware and software, plus fast network connections. New technologies can often be provided as an add-on or plug-in, rather than a replacement. As the new technology become perfected and widely accepted, it may become the new standard. Layered approaches have been successful in the past and they are compelling for accommodating a wide range of users. They are easy to implement when planned in advance, but often difficult to retrofit.

Advocates who promote accommodation of handicapped users often describe the curbcut - a scooped out piece of sidewalk to allow wheel-chair users to cross streets. Adding curbcuts after the curbs have been built is expensive, but building them in advance reduces costs because less material is needed. The benefits extend to baby carriage pushers, delivery service workers, bicyclists, and travelers with roller bags. Other computer-related accommodations that benefit many users are putting the power switch in the front of computers, building adjustable keyboards, and allowing user control over audio volume, screen brightness, and monitor position.

Automobile designers have long understood the benefits of accommodating a wide range of users. They feature adjustable seats, steering wheels, mirrors, and lighting levels as standard equipment and offer optional equipment for those who need additional flexibility. 
Reaching a broad audience is more than a democratic ideal; it makes good business sense. The case for network externalities, the concept that all users benefit from expanded participation (Shapiro and Varian, 1998; Borenstein, 1998), has been made repeatedly. Facilitating access and improving usability expands markets and increases participation of diverse users whose contributions to the community may be valuable to many. Broadening participation is not only an issue of reducing costs for new equipment. As the number of users grows, the capacity to rapidly replace a majority of equipment declines, so strategies that accommodate a wide range of equipment will become even more in demand.

With these concerns in mind, the research agenda for universal usability may provoke many innovations for all users.

3.1 Technology variety: Supporting a broad range of hardware, software, and network access

The first challenge is to deal with the pace of technology change and the variety of equipment that users employ. The stabilizing forces of standard hardware, operating systems, network protocols, file formats, and user interfaces are undermined by the rapid pace of technological change. The technological innovators delight in novelty and improved features. They see competitive advantage to advanced designs, but these changes disrupt efforts to broaden audiences and markets. Since limiting progress is usually an unsatisfactory solution, an appealing strategy is to make information content, online services, entertainment, and user interfaces more malleable or adaptable.

The range of processor speeds in use probably varies by a factor of 1000 or more. Moore's Law, which states that processor speeds double every 18 months, means that after ten years the speed of the newest processors are 100 times faster. Designers who wish to take advantage of new technologies risk excluding users with older machines. Similar changes for RAM and hard disk space also inhibit current designers who wish to reach a wide audience. Other hardware improvements such as increased screen size and improved input devices also threaten to limit access. Research on accommodating varying processor speed, RAM, hard disk, screen size, and input devices could help cope with this challenge.

Another hardware related research topic is software to convert interfaces and information across media or devices. For users who wish to get web page contents read to them over the telephone or for blind users, there are already some services (http://www.conversa.com), but improvements are needed to speed delivery and extract appropriately (Thomas, Basson, and Gardner-Bonneau, 1999). Accommodating assorted input devices, by a universal bus would allow third party developers to create specialized and innovative devices for users with handicaps or special needs (Perry, Macken, Scott, and McKinley, 1997).

Software changes are a second concern. As applications programs mature and operating systems evolve users of current software may find their programs become obsolete because newer 
versions fail to preserve file format compatibility. Some changes are necessary to support new features, but research would be helpful to identify modular designs that promote evolution while ensuring compatibility and bi-directional file conversion. The Java movement is a step in the right direction, since it proposes to support platform independence, but its struggles indicate the difficulty of the problems.

Network access variety is a third problem. Some users will continue to use slower speed (14.4Kbps) dial-up modems while others will use 10Mbps cable modems. This 100-fold speedup requires careful planning to accommodate. Since many web pages contain large amounts of graphics, providing user control of byte counts would be highly advantageous. Most browsers allow users to inhibit graphics, but more flexible strategies are needed. Users should be able to specify that they want reduced byte count graphics, and invoke procedures on the server to compress the image from $300 \mathrm{~K}$ to $100 \mathrm{~K}$ or to $30 \mathrm{~K}$. With additional image analysis research, servers should be able to produce a $10 \mathrm{~K}$ image outline. An alternative is simply a 100 byte textual label, supplied by the author, already a requirement for many web sites that accommodate blind users.

3.2 User diversity: Accommodating users with different skills, knowledge, age, gender, handicaps, literacy, culture, income, etc.

A second challenge to broadening participation is the diversity of users (Kobsa and Stephanidis, 1998; Fink, Kobsa, and Nill, 1999). Since skill levels with computing vary greatly, many search engines provide a basic and advanced dialog box for query formulation. Since knowledge levels in an application domain vary greatly, some sites provide two or more versions. For example, the National Cancer Institute provides thoughtful information on many forms of cancer for patients and more detailed information for physicians. Since children differ from adults in their needs, NASA provides a K-12 (kindergarten through $12^{\text {th }}$ grade) section of many of their space mission pages. Many other examples of accommodating diverse users by simply creating separate sections of a web site can be found. Universities often segment their sites for applicants, current students, or alumni, but then provide links to shared resources of mutual interest.

Similar segmenting strategies can be employed to accommodate users with poor reading skills or users who require other natural languages. While there are some services to automatically convert web pages to multiple languages (http://www.altavista.com, http://www.scn.org/spanish.html) the quality of human translations is much higher. Research on tools to facilitate preparation and updating of web sites in multiple languages would be helpful. For example, if an e-commerce site maintained multiple language versions of a product catalog, then it would be useful to have a tool that facilitated simultaneous changes to a product price (possibly in different currencies), name (possibly in different character sets), or description (possibly tuned to regional variations). A more difficult problem comes in trying to accommodate users with a wide range of incomes, cultures, or religions. Imagine trying to prepare multiple music, food, or clothing catalogs that 
were tuned to local needs by emphasizing highly desired products and eliminating offensive items. E-commerce sites that are successful in these strategies are likely to be more widely used.

Another set of issues deals with the wide range of handicaps, or differential capabilities of users. Many systems allow partially sighted users, especially elderly users, to increase the font size or contrast in documents, but they rarely allow users to improve readability in control panels, help messages, or dialog boxes. Blind users will be more active users of information and communications services if they can receive documents by speech generation or in Braille, and provide input by voice or their customized interfaces. Physically handicapped users will eagerly use services if they can connect their customized interfaces to standard graphical user interfaces, even though they may work at a much slower pace. Cognitively impaired users with mild learning disabilities, dyslexia, poor memory, and other special needs could also be accommodated with modest design changes to improve layouts, control vocabulary, and limit short-term memory demands.

Expert and frequent users also have special needs. Enabling customization that speeds highvolume users, macros to support repeated operations, and inclusion of special-purpose devices could bring many benefits. Research on the needs of high-end users could improve interfaces for all users.

Finally, appropriate services for a broader range of users need to be developed, tested, and refined. Corporate knowledge workers are the primary audience for many contemporary software projects, so the interface and information needs of unemployed, homemakers, disabled, or migrant workers, usually get less attention. This has been an appropriate business decision till now, but as the market broadens and key societal services are provided electronically, the forgotten users must be accommodated. For example, Microsoft Word provides templates for marketing plans and corporate reports, but every citizen interfaces might help with job applications, babysitting cooperatives, or letters to city hall.

The growth of online support communities, medical first-aid guides, neighborhood-improvement councils, and parent-teacher associations will be accelerated as improved interface and information designs are developed. Community-oriented plans for preventing drug or alcohol abuse, domestic violence, or crime could also benefit from research on interface and information design. Such research is especially important for government web sites, since their designers are moving towards providing basic services such as driver registration, business licenses, municipal services, tax filing, and eventually voting. Respect for the differing needs of users will do much to attract them to using advanced technologies.

3.3 Gaps in user knowledge: Bridging the gap between what users know and what they need to know 
A third challenge is to bridge the gap between what users know and what they need to know. A wide variety of strategies is used in practice and competing theories are promoted by researchers, but their efficacy is poorly studied.

Users approach new software tools with diverse backgrounds. Sometimes they need only a few minutes of orientation to understand the novelties and begin to use new tools successfully. Often users need more time to acquire knowledge about the objects and actions in the application domain and in the user interface. To help these users, designers need clear and validated guidance on effective tutorials for novices, lucid instructions for common tasks, constructive help for intermittent users, and compact presentations for experts. Improved designs of user interfaces should emphasize error prevention, but specific, positive tone and constructive error messages, with easy repair strategies, are also important. Other potential aids are easily reversible actions and detailed history keeping for review and consultation with peers and mentors. Systematic logging of usage and observations of users would help greatly. Research on tools to help developers provide, assess, and refine such services would be useful.

A more fundamental interface and information design research problem is how to develop improved strategies for evolutionary learning. Proposals for appropriate layered designs, graceful progressive disclosure, and comprehensible user-controlled options need to be implemented and tested. Could users begin with an interface that contained only basic features (say five percent of the full system) and become experts at this level within a few minutes? Introductions and tutorials could be finely tuned to meet the needs of users of this narrow set of features. Then how might they explore additional features and add them as needed? Similarly, how can users understand and cope with the many exotic options in modern word processors, email handlers, and web browsers? A good beginning has been made with concepts such as layered implementations and the minimal manual (van der Meij and Carroll, 1995), but scaling up and broader application will require further research.

Finally, the provision of online help by way of email, telephone, video conferencing, and shared screens needs further examination and design improvements. There is appealing evidence that online social mechanisms among peers such as news groups, listservs, and frequently asked question (FAQ) lists are helpful, but there is little research that distinguishes among the best and worst of these. While consumer advocates have studied the time to get responses from telephone help desks offered by software providers, there is little insight about how to make these services more effective. Best practices, validated analyses, guidelines, and theories could all be improved through extensive research.

\section{Conclusion}

Attaining the benefits of universal access to web-based and other information, communications, entertainment, and government services will require a more intense commitment to lowering costs, coupled with human-computer interaction research and usability engineering. A starting point for research would be a program that addressed at least these universal usability challenges: 
- Technology variety: Supporting a broad range of hardware, software, and network access

- User diversity: Accommodating users with different skills, knowledge, age, gender, handicaps, literacy, culture, income, etc.

- Gaps in user knowledge: Bridging the gap between what users know and what they need to know

America OnLine claims that "It's so easy to use. That's why we are number one." They recognize the centrality of usability, and have done well to make their services usable by many. Their success is admirable in reaching a fraction of the potential audience, but much work remains to achieve the goal of universal usability. In planning ahead to a time when vital services will be provided online and when novel social, economic, and political programs become possible because of widespread citizen participation, we should consider what research is needed to support our aspirations.

Acknowledgements: Thanks to Alfred Kobsa, Gary Marchionini, Elizabeth Murphy, Jenny Preece, Anne Rose, Andrew Sears, David Silver, Barbara Simons, John Thomas and Bryant York for comments on early versions of this paper. Thanks also to continuing support from IBM and the US Census Bureau.

\section{References}

Anderson, R.H., Bikson, Tora, Law, Sally Ann, and Mitchell, Bridger M., 1995. Universal access to e-mail: Feasibility and societal implications. Santa Monica, CA: The Rand Corporation. URL:http://www.rand.org/publications/MR/MR650/

Bergman, Eric and Johnson, Earl, Towards accessible human-computer interaction, In Nielsen, Jakob (Editor), Advances in Human-Computer Interaction: Volume 5, Ablex Publishing, Norwood, NJ (1995). Also at http://www.sun.com/tech/access/updt.HCI.advance.html

Borenstein, Nathaniel, One Planet, One Net, Many Voices, CPSR Newsletter 16, 1 (Winter 1998) $1,5-8$.

Clement, Andrew and Shade, Leslie Regan, The Access Rainbow: Conceptualizing universal access to the information/ communications infrastructure, Univ. of Toronto, (1999, submitted).

Computer Science and Telecommunications Board (CSTB), National Research Council, More than Screen Deep: Toward an Every-Citizen Interface to the Nation's Information Infrastructure, National Academy Press, Washington, DC, 1997.

Fink, J., Kobsa, A., and Nill, A., Adaptable and adaptive information provision for all users, including disabled and elderly people, To appear in New Review of Hypermedia and Multimedia(1999). http://zeus.gmd.de/ kobsa/papers/1999-NRMH-kobsa.ps 
Glinert, E.P., and York, B.W. Computers and people with disabilities, Communications of the ACM 35, 5 (May 1992), 32-35,.

Kobsa, Alfred and Stephanidis, Constantine, Adaptable and adaptive information access for all users, including disabled and elderly people, Proc.2nd Workshop on Adaptive Hypertext and Hypermedia, ACM HYPERTEXT'98 (1998), http://wwwis.win.tue.nl/ah98/Kobsa.html

Kraut, Robert, Scherlis, William, Mukhopadhyay, Tridas, Manning, Jane, and Kiesler, Sara, The HomeNet field trial of residential Internet services, Communications of the ACM 39, 12 (December 1996), 55-63.

Laux, L. F., McNally, P. R., Paciello, M. G., Vanderheiden, G. C., Designing the World Wide Web for people with disabilities: a user centered design approach, Proc. Assets '96 Conference on Assistive Technologies, ACM, New York, (1996), 94-101.

Newell, A. F. (Editor), Extraordinary Human-Computer Interaction: Interfaces for Users with Disabilities, Cambridge University Press, UK (1995).

National Telecommunications and Information Administration, U. S. Dept. of Commerce, Falling Through the Net: Defining the Digital Divide, Washington, DC (July 1999), http://www.ntia.doc.gov/ntiahome/digitaldivide/

Perry, J., Macken, E., Scott, N., and McKinley, J. L., Disability, Inability and Cyberspace, in Human Values and the Design of Technology, Friedman, B. (Editor), CSLI Publications \& Cambridge University Press (1997), 65-89.

Scholtz, Jean, et al., A research agenda for high performance user interfaces: Useful, Usable, and Universal, ACM Special Interest Group on Computer Human Interaction (SIGCHI), New York (1999, forthcoming).

Shapiro, Carl and Varian, Hal R., Information Rules: A Strategic Guide to the Network Economy, Harvard Business School Press, Boston, MA, 1999.

Shneiderman, B., Designing the User Interface: Strategies for Effective Human-Computer Interaction: Third Edition, Addison-Wesley Publ. Co., Reading, MA (1998),

Shneiderman, B. and Rose, A., Social Impact Statements: Engaging public participation in information technology design, In Friedman, B. (Editor), Human Values and the Design of Computer Technology, CSLI Publications \& Cambridge Univ. Press (1997), 117-133.

Silver, David, Margins in the wires: Looking for race, gender, and sexuality in the Blacksburg Electronic Village, In Kolko, B., Nakamura, L. and Rodman, G., Race in Cyberspace: Politics, Identity, and Cyberspace, Routledge, London (1999, in press). 
Thomas, John C., Basson, Sara, and Gardner-Bonneau, Daryle, Universal design and assistive technology, In Gardner-Bonneau, Daryle (Editor), Human Factors and Voice Interactive Systems, Kluwer Academic Publishers, Boston (1999).

van der Meij, Hans and Carroll, John M., Principles and heuristics in designing minimalist instruction, Technical Communication (Second Quarter 1995), 243-261. 